\title{
Hippocampal Volumetry as a Biomarker for Dementia in People with Low Education
}

\author{
Jaime D. Mondragóna César Celada-Borjab \\ Fernando Barinagarrementeria-Aldatz ${ }^{c}$ Martín Burgos-Jaramillo ${ }^{d}$ \\ Héctor Manuel Barragán-Camposa \\ a Unidad de Resonancia Magnética, Instituto de Neurobiología, UNAM-Campus Juriquilla, \\ Querétaro, ${ }^{b}$ Instituto Nacional de Psiquiatría Ramón de la Fuente Muñiz, Ciudad de México, \\ 'Hospital H+ Querétaro, Querétaro, and d'Lean Six Sigma, Tecnológico de Monterrey-Campus \\ Querétaro, Santiago de Querétaro, Mexico
}

\author{
Key Words \\ Biomarkers · Hispanics · Cognitive assessment - Dementia - Education · Mild cognitive \\ impairment $\cdot$ MRI $\cdot$ Volumetry $\cdot$ MMSE
}

\begin{abstract}
Background/Aims: To evaluate the relationship between hippocampal volume and cognitive decline in patients with dementia due to probable Alzheimer's disease (AD), amnestic mild cognitive impairment (aMCI) and education, and the possible relationship between cognitive reserve and education in this population. Methods: From February 2013 to October 2015, 76 patients ( 25 men, 51 women) were classified according to the NIA-AA diagnostic criteria. We used two 3.0-tesla MRI scanners and performed manual hippocampal volumetry. Results: Twenty-six patients were found to have AD, $20 \mathrm{aMCI}$ and 30 had normal aging (NA). The mean normalized hippocampal volume in age-, sex- and education (years)-matched subjects was $2.38 \pm 0.51 \mathrm{~cm}^{3}$ in $A D(p<0.001), 2.91 \pm 0.78 \mathrm{~cm}^{3}$ in $\mathrm{aMCI}(p=0.019)$ and $3.07 \pm 0.76 \mathrm{~cm}^{3}$ in NA. Conclusion: Psychometric test (MMSE and MoCA) scores had a good to strong positive correlation with statistically significant differences in the entire population and healthy subjects but not among dementia patients and lower educational level groups. The patients with low education had greater hippocampal volumes, which is in line with the cognitive reserve theory; lower-educated individuals can tolerate less neuropathology and will thus show less atrophy at a similar level of cognitive performance than higher-educated subjects.
\end{abstract}


Mondragón et al.: Hippocampal Volumetry as a Biomarker for Dementia in People with Low Education

\section{Introduction}

In 2010, approximately 35.6 million people in the world were living with dementia, of which 54\% lived in low- and middle-income countries [1]. Dementia due to probable Alzheimer's disease (AD) represents between 60 and $80 \%$ of all dementias [2]. According to the World Health Organization (WHO), among noncommunicable diseases (NCDs), AD ranks as the 18th disease worldwide with a total of 9,243,325 in people over the age of 60 [3]. According to the same report, AD is 14th among NCDs and 9th among chronic degenerative diseases in the Americas, accounting for a total of 1,920,390 cases in patients over 60 years. It is prevalent in populations with a bulb distribution; however, it is the fourth leading cause of disability-adjusted life years in countries with high per capita income [3].

The total number of estimated AD cases worldwide by 2030 is 65.7 million and 115.4 million by 2050; this represents a twofold population increase in the next 20 years [2]. The Latin American population is suffering the most drastic changes within this group, expecting an increase in the population of $77-146 \%$ over the next 20 years; this represents the highest increase in incidence among all regions of the world in this period [2]. The age-specific prevalence of dementia varies little between world regions and possibly even overlaps [4]. In 2010, 4.7 million people, 65 and older, lived with AD in the US. By 2050, the population over 65 with $\mathrm{AD}$ is projected to increase to 13.8 million, of which about 7 million will be over 85 years of age [5]. The total estimated worldwide cost for dementia in 2010 was USD 604 billion, representing about $1 \%$ of the global gross domestic product [1]. By 2030, the total cost for the social care of dementias is projected to increase by $85 \%$; which amounts to approximately USD 1.117 trillion. [1] The 2009 World Alzheimer Report predicts that the prevalence of this disease will increase between 134 and $146 \%$ by 2030 in Latin America [6]. Traditionally, the clinical approach to the diagnosis of dementia is by steps; first, recognition of dementia syndrome is required, followed by determination of its cause [7]. The Diagnostic and Statistical Manual of Mental Disorders, fourth edition (DSM-IV), and the criteria published by the National Institute of Neurological and Communicative Disorders and Stroke and the Alzheimer's Disease and Related Disorders Association (NINCDS-ADRDA) are the most accepted clinical criteria for AD [7]. It is a progressive brain disease defined by a clinical and a pathological component. The recently published criteria by the National Institute on AgingAlzheimer's Association (NIA-AA) have two tasks at hand: to establish clinical diagnosis and create a clinical research classification [8-11]. The main criteria for the diagnosis of AD include ruling out neuropsychiatric, cognitive and behavioral symptoms that: (a) interfere with the capacity to perform work or daily-life activities; (b) contribute to performance decline; (c) are not explained by delirium or another psychiatric disorder [8]. Cognitive disability is diagnosed through a complete patient anamnesis, obtained from the patient and the primary caretaker, and corroborated by objective cognitive assessment (e.g. mental status examination or neuropsychological testing] [8]. Lastly, cognitive impairment involves at least two of the following cognitive domains: (a) ability to acquire and remember new information; (b) reasoning ability and judgment; (c) visual and spatial skills; (d) language functions; and (e) personality changes [8]. According to this classification, AD is classified as: (a) dementia due to probable AD; (b) dementia due to possible $\mathrm{AD}$; and (c) dementia due to probable or possible $\mathrm{AD}$ with pathophysiological evidence of $\mathrm{AD}$ process [8]. The difference between the $\mathrm{AD}$ and amnestic mild cognitive impairment (aMCI) definition lies in the determination of the inability to function in the workplace or everyday activities [8]. Mild cognitive impairment (MCI) is the transitional cognitive state between normal aging (NA) and mild dementia [12]. Two presentations of MCI are described; however, aMCI is of particular interest due to its emphasis on memory loss. Diagnostic criteria for aMCI are the following: (a) complaints of memory loss, corroborated by the primary caretaker; (b) memory disorder for age and 
Mondragón et al.: Hippocampal Volumetry as a Biomarker for Dementia in People with Low Education

education; (c) general cognitive function preserved; (d) ability to perform daily activities; and (e) without dementia [12]. Aging is a continuous physiological phenomenon where the body's adaptive capabilities come into play until they stop functioning, resulting in a decline in organ function, followed by a decline of the tissues and the general body appearance [13]. All physiological systems age at a certain pace with varying consequences depending on the organ. Meanwhile, the concept of optimal aging has replaced the concept of NA, which can be enhanced by adjusting lifestyle measures [13].

With the advent of new diagnostic tools, the need to update and expand the diagnostic criteria of $\mathrm{AD}$ and $\mathrm{aMCI}$ has emerged; these new diagnostic criteria must include both structural and functional damage measuring tools [7]. Biomarkers are physiological, biochemical and anatomical parameters that can be measured in vivo and reflect the pathophysiological processes associated with a disease [11]. To date, only five biomarkers have been incorporated into the diagnostic criteria for clinical research in AD: (a) $A \beta_{42}$ protein cerebrospinal fluid levels; (b) positron emission tomography (PET) with $\beta$-amyloid radiotracer, such as Pittsburg compound (C-PIB) or 2-(1-\{6-[(2-[F]fluoroethyl)(methyl)amino]-2-naphtyl\}ethylidene) malono nitrile $\left({ }^{18} \mathrm{~F}-\mathrm{FDNNP}\right)$; (c) tau protein cerebrospinal fluid levels; (d) structural MRI; (e) PET using fluorodeoxyglucose $\left({ }^{18} \mathrm{~F}\right.$-FDG) $[11,14]$. No serum biomarkers have been validated to this day for the diagnosis of $\mathrm{AD}$; while imaging biomarkers validated for clinical research include MRI and PET [11]. For the time being, as far as genetic markers are concerned, there are three with a clear association and one considered a risk factor: APP, PSEN1, PSEN2

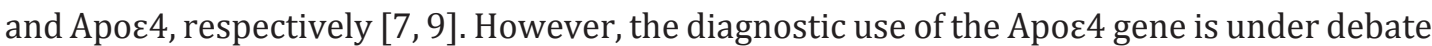
for its use in clinical research [8]. Similar to the diagnostic criteria established for AD by the NIA-AA, the criteria for aMCI consist of two groups: clinical diagnosis and clinical research criteria [10]. In contrast to clinical criteria, clinical research criteria incorporate the use of biomarkers; this is due to the existing limitations on these tools for clinical diagnosis. These limitations include the appropriate use of these markers, standardization of procedures for the use of these markers, the appropriate allocation of breakpoints to establish the diagnosis, and accessibility to markers may be limited to certain environments [10]. Currently, the use of biomarkers as clinical diagnostic tools for $\mathrm{AD}$ and $\mathrm{aMCI}$ is not recommended due to the lack of evidence and standardization of the cutoff points of diagnostic thresholds $[8,10]$. The aim of this study was to evaluate the relationship between hippocampal volume and cognitive decline between $\mathrm{AD}$, aMCI and NA in people with low education and the possible relationship between cognitive reserve and education in this unique population. This study attempts to provide information about an understudied group, low educated individuals, in order to further understand the theory of cognitive reserve.

\section{Materials and Methods}

Seventy-six patients were included from February 2013 to October 2015. All patients and control subjects were referred by a specialist (geriatrician, neurologist, psychiatrist) with experience in dementia diagnosis. The subjects were classified according to the diagnostic criteria of the NIA-AA and psychometric tests, such as Mini-Mental State Examination (MMSE) and the Montreal cognitive assessment (MoCA), as part of a comprehensive assessment of the patient. We conducted an observational study with three groups of adults over 60 years old: (1) patients with dementia due to probable AD; (2) patients with aMCI; and (3) a control group with subjects with NA. Patients referred with a diagnosis of cognitive impairment from various public institutions and private practice, from 13 states across Mexico to the Magnetic Resonance Unit at the Instituto de Nurobiología, Universidad Nacional Autónoma de México (UNAM), were selected to participate. Informed consent was obtained for each patient and 

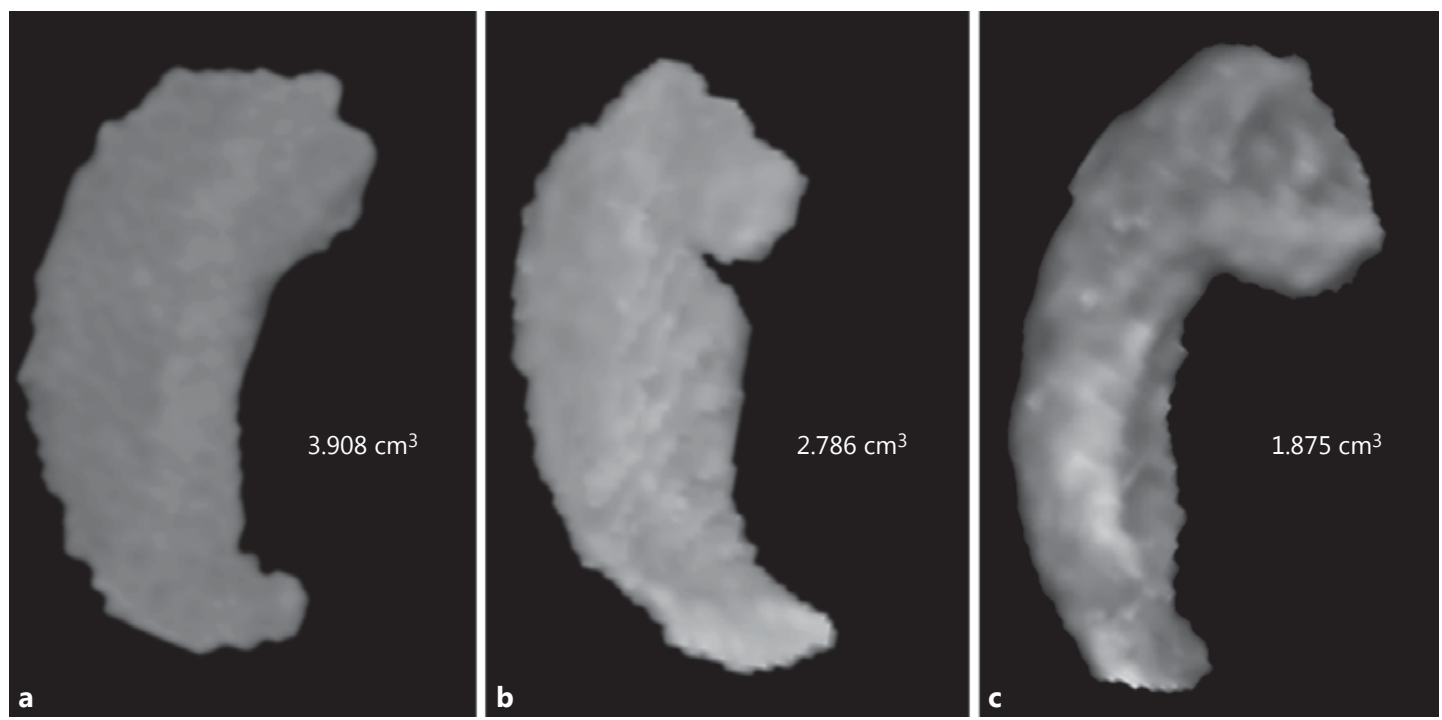

Fig. 1. Reconstruction of hippocampal volumes. 3D reconstruction image of the right hippocampi in patients with NA (a), aMCI (b) and dementia due to probable AD (c).

control subject. The control subjects were chosen from patients undergoing a non-brain related magnetic resonance (MR) and showed no structural lesions. The project was approved by the bioethical committee of the Instituto de Neurobiología, UNAM-Campus Juriquilla.

We began with anamnesis of the patient or control subject; the medical history registered included: marital status, education, previous occupation, family history of dementia, comorbidities and handedness. Following the subject's interview, the information previously registered was corroborated with the informant or primary caretaker; an informant or primary caretaker was always interviewed, including in the control group. As the informant was being interviewed, the patient or control subject took the MMSE and the MoCA psychometric tests. We utilized the psychometric test cutoff points for dementia of 23 for the MMSE and of 26 for the MoCA as suggested by authors $[15,16]$. Control subjects with lower scores were included as long as clinically they were not demented and had lower education. Psychological and cognitive assessments were performed by a physician ascribed to the Magnetic Resonance Unit. MR images were acquired using a 3.0-tesla MR scanner, incorporating the structural sequences: T1; T2; fluid-attenuated inversion recovery (FLAIR); echo gradient; 3D time of flight (3D TOF); along with a three-dimensional spoiled gradient recalled acquisition in steady state (3D SPGR) sequence and axial T1 fast field echo (AxT1FFE) sequence. The three-dimensional sequences used in the manual measurement of both hippocampi were reconstructed using the AW Volumeshare 2.0 software (General Electric, Milwaukee, Wis., USA) while using atlases and manual volumetric protocols previously described [17-23]. After the hippocampal reconstruction, the images were saved in a database for their subsequent analysis (fig. 1). The raters (J.D.M. and C.C.-B.) were blind to the diagnosis and psychometric tests of each subject. Subsequently, the total brain volume was measured using the previously described technique [24]. To correct for MR scanner variability, the images were aligned using the anterior and posterior commissure based on the Talairach coordinate system; while the intensities were adjusted and the volumes were measured at the same workstation, using the program Volumeshare 2.0 from GE. The intracranial volume was measured using the technique described by Colliot et al. [25]. Interpretation of the images was performed by a 
Mondragón et al.: Hippocampal Volumetry as a Biomarker for Dementia in People with Low Education

neuroradiologist (H.M.B.-C.) to exclude comorbidities through the following sequence analysis: sagittal T1, axial T1, T2, FLAIR; gradient echo; coronal T2 and 3D TOF.

The inclusion criteria in this study were the following: (a) subjects 60 years or older; (b) patients referred for dementia, cognitive impairment, cognitive decline, AD, memory loss and MCI; (c) patients with clinical cognitive decline symptoms; (d) patients that accepted to participate in this study and signed an informed written consent; and (e) patients with established dementia diagnosis. The exclusion criteria were the following: (a) refusal to participate; (b) patients with permanent or transitory amnesic syndromes such as Korsakoff's syndrome, paraxial amnesic syndromes (thalamic vascular lesions and rupture of the anterior communicating artery), amnesic ictus and transient epileptic amnesia; (c) patients with cognitive decline secondary to organic pathology such as Parkinson's disease, Lewy bodies dementia, frontotemporal dementia (Pick's disease), vascular dementias, Huntington's disease, progressive supranuclear palsy (Steele-Richardson-Olszewski syndrome), corticobasal degeneration, normal pressure hydrocephalus (Hakim-Adams syndrome) and focal cortical atrophy.

For image acquisition in the hippocampal volumetric reconstruction, T1 MRI sequences were used; these consisted of 128 axial slices parallel adjacent to the anteroposterior commissure line, with 1.2-mm thickness between each slice and an echo gradient (3D SPGR and axial 3D FFE) sequence (repetition time/echo time, 7.8/2.4 ms; FOV, $24 \times 12 \mathrm{~cm}$; and matrix, $256 \times 192$ ). All images were acquired on 3.0-tesla MR scanners (Wisconsin and Philips Achieva 3.0T TX, Philips Medical Systems, Best, The Netherlands, and Discovery General Electric 3.0T MR750, General Electric, Milwaukee, Wis., USA).

Acceptance for structural volumetry as a diagnostic tool for clinical research in aMCI and AD includes the hippocampal and entorhinal regions [26-28]. According to the Alzheimer's Association Neuroimaging Work Group Consensus Report, the following recommendations are provided to decrease the volumetric protocol limitations: (a) image parameter standardization, whenever possible; (b) use of a widely accepted protocol for the interpretation of medial temporal atrophy in MR; and (c) the need for prospective studies to evaluate the usefulness of MR [29]. Most hippocampal segmentation errors are found in the head and tail, along with the anterior boundary of the amygdala [30]. In general, automatic hippocampal segmentation overestimates the volume compared with manual segmentation [29].

Hippocampal volume normalization was performed using the total intracranial volume, following the previously described definition [24], where the normalized hippocampal volume (NHV) is equal to the population's mean total intracranial volume (mTIV) multiplied by the observed hippocampal volume (HV), divided by the individual's total intracranial volume (TIV):

$$
\mathrm{NHV}=(\mathrm{mTIV} \times \mathrm{HV}) / \mathrm{TIV} .
$$

Normalization of the total brain volume (TBV) was done using the previously described definition [23,31], where the normalized total brain volume (NTBV) is equal to the observed total brain volume minus the product between the regression line coefficient of the normative volume/total brain volume regression line $(B=0.397)$ multiplied by the difference between total intracranial volume and the mean total intracranial volume (mTBV) of the population. The regression line coefficient was calculated by plotting the normative volume versus the total brain volume, and the slope of the regression line yielded B:

$$
\text { NTBV }=\text { TBV }- \text { B(TIV }- \text { mTBV }) \text {. }
$$

Statistical analysis was performed using IBM SPSS Statistics 20 (IBM, Armonk, N.Y., USA). We used the nonparametric test, Mann-Whitney U test, for independent groups, with which we calculated the statistical values for $\mathrm{p}$ at a confidence interval of $95 \%$. For statistical $\mathrm{p}$ 
Mondragón et al.: Hippocampal Volumetry as a Biomarker for Dementia in People with Low Education

Table 1. Demographic data of the population according to diagnostic group

\begin{tabular}{llllll}
\hline Diagnostic group & $\mathrm{n}$ & Mean age, years & MMSE & MoCA & Education, years \\
\hline AD & 26 & $78.1(60-93)$ & $17.7(11-23)$ & $12.5(3-24)$ & $7.4(0-18)$ \\
aMCI & 20 & $74.5(62-93)$ & $26.2(20-30)$ & $24.0(17-29)$ & $9.0(0-22)$ \\
NA & 30 & $71.5(60-87)$ & $27.5(20-30)$ & $25.1(15-30)$ & $9.5(0-22)$ \\
Population & 76 & 74.6 & 23.8 & 20.5 & $8.7(0-22)$ \\
\hline
\end{tabular}

$\mathrm{n}=$ Number; MMSE = Mini-Mental State Examination; MoCA = Montreal cognitive assessment; $\mathrm{AD}=$ dementia due to probable Alzheimer's disease; aMCI = amnestic mild cognitive impairment; $\mathrm{NA}=$ normal aging. Figures in parentheses indicate range.

values within two groups, with a total population greater than 30 subjects, the independentsamples t test was performed to compare means. Analysis of variance (ANOVA) for one factor was performed to calculate the $\mathrm{p}$ values between all three groups, and analysis of covariance was performed to compare two measurable variables while excluding a nominal variable. The Bonferoni post hoc test was used to adjust $\mathrm{p}$ values. Pearson's correlation values were also calculated to assess the association between education and hippocampal volume.

\section{Results}

From February 2013 to October 2015, 76 subjects were recruited to participate in this study, of which the following demographic data are reported (table 1): 25 males, 51 females; 26 with AD, 20 with aMCI and 30 with NA; mean age of the population, 74.6 years (range, 60-93); mean age of patients with $\mathrm{AD}, 78.1$ years (range, 60-93); mean age of patients with aMCI, 74.5 years (range, 62-93); mean age of subjects in the control group, 71.5 years (range, 60-87). Regarding the results of the psychometric tests, the average scores for the MMSE within each group were: 17.7 points (range, 11-23) for the AD group; 26.2 points (range, 20-30) for the aMCI group; and 27.5 points (range, 20-30) for the control group. The results obtained for the MoCA psychometric test were the following: 12.5 points (range, 3-24) for the AD group; 24.0 points (range, 17-29) for the aMCI group; and 25.1 points (range, 15-30) for the control group. The average education for the entire group was 8.7 years (range, $0-22$ ), 7.4 years (range, $0-18$ ) for the AD group, 9-0 years (range, $0-22$ ) for the aMCI group and 9.5 years (range, $0-22$ ) for the NA group.

The psychometric test results according to education group were the following: 22.1 points (range, 13-30) and 14.3 points (range, 3-29) in the MMSE and MoCA, respectively, for the group with three or fewer years of education; 24.3 points (range, 11-30) and 22.8 points (range, 8-29) in the MMSE and MoCA, respectively, for the group with education between 4 and 11 years; and 24.5 points (range, 11-29) and 22.9 points (range, 7-30) in the MMSE and MoCA, respectively, for the group with more than 12 years of education.

The observed hippocampal volumes are volumes measured without normalization to the population's mean or adjusted to the intracranial volume of each patient. The observed mean hippocampal volumes for each group were: $2.34 \pm 0.53 \mathrm{~cm}^{3}$ in $\mathrm{AD}(\mathrm{p}<0.001) ; 2.80 \pm 0.80 \mathrm{~cm}^{3}$ in aMCI ( $\mathrm{p}=0.007) ; 3.04 \pm 0.64 \mathrm{~cm}^{3}$ in NA. The observed right hippocampal volumes for each group were: $2.44 \pm 0.57 \mathrm{~cm}^{3}$ in $\mathrm{AD}(\mathrm{p}<0.001) ; 2.84 \pm 0.89 \mathrm{~cm}^{3}$ in aMCI $(\mathrm{p}=0.007) ; 3.15 \pm$ $0.67 \mathrm{~cm}^{3}$ in NA ( $p<0.001 \mathrm{vs}$. AD). Meanwhile, the observed left hippocampal volumes for each 
Mondragón et al.: Hippocampal Volumetry as a Biomarker for Dementia in People with Low Education

Table 2. Observed and normalized hippocampal volume according to diagnostic group

\begin{tabular}{lllllrr}
\hline Diagnostic group & $\mathrm{HV}, \mathrm{cm}^{3}$ & $\mathrm{p}$ & $\mathrm{NHV}, \mathrm{cm}^{3}$ & $\mathrm{p}$ & $\mathrm{mNHV}, \mathrm{cm}^{3}$ & $\mathrm{p}$ \\
\hline AD & $2.34 \pm 0.53$ & $<0.001$ & $2.37 \pm 0.50$ & $<0.001$ & $2.38 \pm 0.51$ & $<0.001$ \\
aMCI & $2.80 \pm 0.80$ & 0.007 & $2.80 \pm 0.79$ & 0.002 & $2.91 \pm 0.78$ & 0.019 \\
NA & $3.04 \pm 0.64$ & & $3.05 \pm 0.66$ & & $3.07 \pm 0.76$ & \\
\hline
\end{tabular}

$\mathrm{HV}=$ Mean hippocampal volume; NHV= mean normalized hippocampal volume; $\mathrm{mNHV}=$ mean normalized hippocampal volume matched by sex, age and years of education; $\mathrm{AD}=$ dementia due to probable Alzheimer's disease; $\mathrm{aMCI}=$ amnestic mild cognitive impairment; $\mathrm{NA}=$ normal aging.

Table 3. Matched normalized mean hippocampal volume and normalized total brain volume according to diagnostic group

\begin{tabular}{llrrr}
\hline Diagnostic group & $\mathrm{mNHV}, \mathrm{cm}^{3}$ & $\mathrm{p}$ & $\mathrm{NTBV}, \mathrm{cm}^{3}$ & $\mathrm{p}$ \\
\hline AD & $2.38 \pm 0.51$ & $<0.001$ & $994.34 \pm 64.88$ & 0.025 \\
aMCI & $2.91 \pm 0.78$ & 0.019 & $1,066.69 \pm 83.49$ & 0.452 \\
NA & $3.07 \pm 0.76$ & & $1,020.54 \pm 73.20$ & \\
\hline
\end{tabular}

mNHV = Mean normalized hippocampal volume matched by sex, age and years of education; NTBV = normalized total brain volume; $\mathrm{AD}=$ dementia due to probable Alzheimer's disease; $\mathrm{aMCI}=$ amnestic mild cognitive impairment; NA = normal aging.

group were: $2.20 \pm 0.66 \mathrm{~cm}^{3}$ in $\mathrm{AD}(\mathrm{p}<0.001) ; 2.76 \pm 0.76 \mathrm{~cm}^{3}$ in $\mathrm{aMCI}(\mathrm{p}=0.007) ; 2.93 \pm$ $0.64 \mathrm{~cm}^{3}$ in NA.

The mean hippocampal volumes were normalized according to the previously described method [24]; subsequently, the following mean NHVs for each group were obtained: $2.37 \pm$ $0.50 \mathrm{~cm}^{3}$ in $\mathrm{AD}(\mathrm{p}<0.001) ; 2.80 \pm 0.79 \mathrm{~cm}^{3}$ in aMCI ( $\left.\mathrm{p}=0.002\right) ; 3.05 \pm 0.66 \mathrm{~cm}^{3}$ in NA (table 2). Lastly, the normalized total brain volume for each group was: $994.34 \pm 64.88 \mathrm{~cm}^{3}$ in AD (p = $0.025) ; 1,066.69 \pm 83.49 \mathrm{~cm}^{3}$ in aMCI ( $\left.\mathrm{p}=0.452\right) ; 1,020.54 \pm 73.2 \mathrm{~cm}^{3}$ in NA (table 3 ). When the subjects were matched for sex, age and years of education, the mean NHVs were: $2.38 \pm$ $0.51 \mathrm{~cm}^{3}(\mathrm{p}<0.001)$ for $\mathrm{AD} ; 2.91 \pm 0.78 \mathrm{~cm}^{3}(\mathrm{p}=0.019)$ for aMCI; and $3.07 \pm 0.76 \mathrm{~cm}^{3}$ for NA (tables 2 and 3 ).

Pearson correlations were performed comparing mean NHV with years of education and psychometric tests (MMSE and MoCA) in the entire population: the three diagnostic groups included in this study (matched by sex, age and education), as well as to cohorts grouped by level of education (low, intermediate and high) (table 4). Both psychometric tests had a good positive correlation with statistically significant differences in the entire population, while a strong positive correlation with statistically significant differences in the NA diagnostic group. The correlation between MMSE scores and the mean NHV in the entire population was good and positive ( $\mathrm{r}=0.4169)$, as well as with statistically significant differences ( $\mathrm{p}=$ $0.000179)$; while it had a strong positive correlation $(r=0.5062)$ with statistically significant differences ( $p=0.00265$ ) among subjects with NA. As to the correlation between MoCA and the mean NHV, it was good and positive $(r=0.3648)$ with statistically significant differences $(\mathrm{p}=0.001195)$ in the entire population, while it was strong and positive $(r=0.5896)$ with statistically significant differences $(p=0.000305)$ in the NA subgroup. Although the sample size of the population is too small, and within this group subjects differ in clinical status, Pearson correlations were performed in order to further assess the association between 
Table 4. Pearson correlations

\begin{tabular}{lcll}
\hline Correlation & $\mathrm{r}$ & $\mathrm{p}$ & Interpretation \\
\hline $\begin{array}{l}\text { Education and mNHV in } \\
\text { TP }\end{array}$ & 0.0423 & 0.717 & \\
AD & -0.2005 & 0.372 & Insignificant correlation \\
aMCI & 0.002 & 0.994 & Negative weak correlation; not statistically significant differences \\
NA & 0.168 & 0.350 & Insignificant correlation \\
MMSE and mNHV in & & Positive weak correlation; not statistically significant differences \\
TP & 0.4169 & 0.000179 & Good positive correlation with statistically significant differences \\
AD & 0.0935 & 0.678974 & Insignificant \\
aMCI & 0.2702 & 0.27819 & Weak positive correlation; not statistically significant differences \\
NA & 0.5062 & 0.00265 & Strong positive correlation with statistically significant differences \\
MoCA and mNHV in & & & \\
TP & 0.3648 & 0.001195 & Good positive correlation with statistically significant differences \\
AD & -0.0544 & 0.809988 & Insignificant correlation \\
aMCI & -0.3292 & 0.197247 & Good negative correlation; not statistically significant differences \\
NA & 0.5896 & 0.000305 & Strong positive correlation with statistically significant differences \\
MMSE and mNHV in LE & 0.3517 & 0.11795 & Good positive correlation; not statistically significant differences \\
MoCA and mNHV in LE & 0.2837 & 0.212665 & Weak positive correlation; not statistically significant differences \\
MMSE and mNHV in IE & 0.3033 & 0.170019 & Good positive correlation; not statistically significant differences \\
MoCA and mNHV in IE & 0.2783 & 0.209805 & Weak positive correlation; not statistically significant differences \\
MMSE and mNHV in HE & 0.5739 & 0.000479 & Strong positive correlation with statistically significant differences \\
MoCA and mNHV in HE & 0.5963 & 0.00025 & Strong positive correlation with statistically significant differences \\
\hline
\end{tabular}

$\mathrm{r}$ = Pearson's correlation value; mNHV = mean normalized hippocampal volume matched by sex, age and years of education; $\mathrm{TP}$ = total population; $\mathrm{AD}=$ dementia due to probable Alzheimer's disease; $\mathrm{aMCI}=$ amnestic mild cognitive impairment; $\mathrm{NA}=$ normal aging; MMSE = Mini-Mental State Examination; MoCA = Montreal cognitive assessment; LE = low educational level (3 years or fewer); IE = intermediate educational level (between 4 and 11 years); HE = high educational level (more than 12 years).

educational level and hippocampal volume. When the population was subgrouped by educational levels regardless of clinical diagnosis, the correlation between both psychometric tests and the mean NHV was strong and positive with statistically significant differences in the high education cohort. The correlation between the MMSE score and the mean NHV was strong and positive ( $r=0.5739)$ with statistically significant differences $(p=0.000479)$ in the high education cohort; while the correlation between the MoCA score and the mean NHV was strong and positive $(r=0.5963)$ with statistically significant differences $(p=0.00025)$ in this same group. While the correlation between years of education compared to the volume of the hippocampus was weak to insignificant with no statistically significant differences, both psychometric tests correlated with the hippocampal volume in people with a high educational level (more than 12 years), hence allowing these two diagnostic tools to adequately classify dementia patients in the Mexican population with higher education. The good to strong correlation between both psychometric tests and hippocampal volume in the entire population validates its usefulness in the diagnosis of dementia in a population with a heterogeneous educational level; nonetheless, these diagnostic tools have their limitations when it comes to finding a direct correlation between hippocampal volume and the psychometric tests primarily used to diagnose dementia and $\mathrm{MCI}$ in populations with low and intermediate educational levels.

When the subjects were grouped by educational level cohorts independent of the diagnosis [(1) 3 years or fewer, (2) between 4 and 11 years and (3) more than 12 years] the following results were obtained for the mean NHV ( $p$ values were calculated using one-way ANOVA, when comparing one diagnostic group with the other two). The mean NHV for each 
Mondragón et al.: Hippocampal Volumetry as a Biomarker for Dementia in People with Low Education

group was: $2.72 \pm 0.68 \mathrm{~cm}^{3}$ for subjects with 3 or fewer years of education ( $\left.\mathrm{p}=0.51\right) ; 2.67 \pm$ $0.72 \mathrm{~cm}^{3}$ for the subjects with total educational years between 4 and 11 years $(p=0.33)$; and $2.83 \pm 0.73 \mathrm{~cm}^{3}$ for the subjects with 12 or more educational years $(\mathrm{p}=0.41)$. While the normalized total cerebral volume for these same three groups was: $1,019.17 \pm 68.57 \mathrm{~cm}^{3}$ for subjects with 3 or fewer years of education ( $p=0.34) ; 1,028.32 \pm 87.92 \mathrm{~cm}^{3}$ for the subjects with total educational years between 4 and 11 years $(p=0.27)$; and $1,025.47 \pm 79.40 \mathrm{~cm}^{3}$ for the subjects with 12 or more educational years $(p=0.63)$. None of the volumetric measurements had statistically significant differences when grouped by educational level cohorts.

The following variables were analyzed using one-way ANOVA, in search of statistically significant differences between the mean hippocampal volume of the subgroups derived from the nominal definition of each variable: (1) sex, subdivided in male and female; (2) age group, subdivided into 65-74, 75-84 and 85 and older; (3) body mass index, subdivided into <19, 19-24.99, 25-29.99, 30-34.99, 35-39.99, >40; (4) marital status, subdivided in married, not married, never married; (5) previous occupation, subdivided in those that primarily involved physical performance and those that primarily involved mental performance; (6) presence or absence of family history of dementias; (7) comorbidities, subdivided into those characteristic of the metabolic syndrome (defined by the American Heart Association), other diseases and no comorbidities; (8) handedness, subdivided into right, left and ambidextrous; (9) education, subdivided into 3 or fewer years, between 4 and 11 years, and 12 years or more; and lastly (10) MR scanner used subdivided into GE or Philips. Differences were considered statistically significant with a p value $\leq 0.05$; however, none of the aforementioned variables showed statistically significant differences when comparing the volumes previously described.

\section{Discussion}

The future in the diagnosis of $\mathrm{AD}$ and $\mathrm{aMCI}$ will continue to be based on clinical data; nonetheless, biomarkers will continue to expand the diagnostic frontier. To date, biomarkers have only been accepted for the use in the classification in clinical criteria; nonetheless, there are currently various efforts to standardize and set the limits and cutoff points for AD, aMCI and NA [9-11]. To date, only structural measurements, hippocampal and entorhinal volumetry, are accepted among diagnostic criteria in clinical research [11]. Hippocampal volumetry is well accepted as a clinical research diagnostic tool for early detection of AD and aMCI [28]. There is a correlation between hippocampal atrophy and histological findings of neuronal loss and severity of the pathology in $\mathrm{AD}$ [32]. There is also a correlation between hippocampal hypotrophy and cognitive impairment [32]. Although volumetric assessment serves to stage the patient's cognitive deficits, the rate of atrophy is the most useful index to determine disease progression [24, 33].

An effort to standardize hippocampal volumes is under way. However, hippocampal volumes $>3 \mathrm{~cm}^{3}$ are considered normal; meanwhile, volumes $<2 \mathrm{~cm}^{3}$ are considered abnormal $[20,24]$. Studies report average volumes of $3.267 \mathrm{~cm}^{3}$ [20] and $3.244 \mathrm{~cm}^{3}$ [21] as normal in healthy population with a mean age of $40 \pm 13$ years (range, 24-42 years). Several groups have reported mean NHVs of $2.436-2.860 \mathrm{~cm}^{3}$ in subjects with NA, of $1.993-2.557 \mathrm{~cm}^{3}$ in patients with aMCI and $1.804-2.160 \mathrm{~cm}^{3}$ in patients with AD [24, 34, 35]. Boccardi et al. [33] reported mean hippocampal volumes normalized to the intracranial volume of $2.436 \mathrm{~cm}^{3}$ in subjects with NA, average education of 16 years and mean age of 76 years; $1.993 \mathrm{~cm}^{3}$ in patients with aMCI, average education of 16 years and mean age of 76 years; $1.804 \mathrm{~cm}^{3}$ in patients with $\mathrm{AD}$, average education of 15 years and mean age of 76 years.

If the population obtained from the Alzheimer's Disease Neuroimaging Initiative (ADNI), described by Boccardi et al. [33], is compared with the Mexican population in this study, the 
Mondragón et al.: Hippocampal Volumetry as a Biomarker for Dementia in People with Low Education

Table 5. Comparison between the Turkish and the Mexican population

\begin{tabular}{lllllll}
\hline Diagnostic group & $\mathrm{n}$ & $\begin{array}{l}\text { Mean age, } \\
\text { years }\end{array}$ & $\begin{array}{l}\text { MMSE, } \\
\text { points }\end{array}$ & $\begin{array}{l}\text { Education, } \\
\text { years }\end{array}$ & $\begin{array}{l}\text { NHV, } \\
\mathrm{cm}^{3}\end{array}$ & $\begin{array}{l}\text { Population } \\
\text { differences }\end{array}$ \\
\hline AD & 26 & 78.1 & 17.7 & 7.4 & 2.37 & $+0.14 \mathrm{~cm}^{3}$ \\
AD (Turkish) & 26 & 73.9 & 21.4 & 5.0 & 2.16 & $-4.67 \%$ \\
aMCI & 20 & 74.5 & 26.2 & 9.0 & 2.80 & $+0.24 \mathrm{~cm}^{3}$ \\
aMCI (Turkish) & 22 & 71.3 & 26.6 & 8.0 & 2.56 & $-8.57 \%$ \\
NA & 30 & 71.5 & 27.5 & 9.5 & 3.00 & $+0.21 \mathrm{~cm}^{3}$ \\
NA (Turkish) & 15 & 70.8 & 28.9 & 9.5 & 2.86 & $-8.86 \%$ \\
\hline
\end{tabular}

$\mathrm{n}$ = Number; MMSE = Mini-Mental State Examination; MoCA = Montreal cognitive assessment; NHV = mean normalized hippocampal volume; $\mathrm{AD}=$ dementia due to probable Alzheimer's disease; $\mathrm{aMCI}=$ amnestic mild cognitive impairment; NA = normal aging.

mean NHVs are higher in the Mexican group. The ratios between these two groups are the following: $2.436-3.693 \mathrm{~cm}^{3}$ in subjects with NA; $1.993-2.887 \mathrm{~cm}^{3}$ in patients with aMCI; and $1.804-2.411 \mathrm{~cm}^{3}$ in patients with AD. This volume difference phenomenon can be explained by the cognitive reserve concept. The concept of cognitive reserve arises from the need to explain the association between positive findings in the various diagnostic tests for AD in patients without clinical disease [36]; making the association between more education and greater ability to tolerate pathological burden [37, 37], hippocampal atrophy in this case.

It is suitable to explore the volumetric similarities and differences of two populations with similar education, as this analysis gives insight about possible cutoff points among groups with low education. This evaluation is possible by comparing the results obtained by this study and Turkish research groups. Yavuz et al. [35] reported mean NHVs of $2.858 \mathrm{~cm}^{3}$ in subjects with NA, average educational level of 9.5 years, mean age of 70.8 years and average MMSE score of 28.9 points; $2.557 \mathrm{~cm}^{3}$ in patients with aMCI, average education of 8 years, mean age of 71.3 years and average MMSE score of 26.6 points; and $2.160 \mathrm{~cm}^{3}$ in patients with $\mathrm{AD}$, average education of 5 years, mean age of 73.9 years and average MMSE score of 21.4 points. When comparing the mean NHVs of the Turkish population with the Mexican population in this study, we observed that the volumes of the Mexican population are higher at a ratio of: $2.858-3.693 \mathrm{~cm}^{3}$ in subjects with $\mathrm{NA} ; 2.557-2.887 \mathrm{~cm}^{3}$ in patients with aMCI; and $2.160-2.411 \mathrm{~cm}^{3}$ to in patients with AD (table 5).

Unlike the population studied in Turkey, the volume of the control group in the Mexican population was within accepted parameters of normality. The aMCI volumes in the Mexican population are higher by $0.310 \mathrm{~cm}^{3}$, equivalent to $11.35 \%$, appearing within the ambiguous diagnostic boundaries between $\mathrm{AD}$ and NA in the $2-3 \mathrm{~cm}^{3}$ range. These volume differences can be explained by anatomical variations of the subjects and the heterogeneity of the sample, since the samples are demographically almost identical, with the exception of age, where the Mexican population has a higher average. The AD volumes in the Mexican population were again higher by $0.251 \mathrm{~cm}^{3}$, equivalent to $10.98 \%$. These differences are lower than the two previous groups discussed; however, the Mexican population studied had a higher mean age and a greater cognitive impairment quantified by the MSSE scores than the Turkish population. Although the Mexican and Turkish populations have different reported volumes, these changes can be explained by anatomical variations and fall within acceptable ranges, reported in the literature. All things considered, tentative cutoff points can be set by integrating the information from both of the aforementioned populations; nevertheless, more data are needed to validate or discredit these ranges. 
Mondragón et al.: Hippocampal Volumetry as a Biomarker for Dementia in People with Low Education

The normalized total brain volume is reported in this study in order to provide a reference point about the generalized atrophy present in $\mathrm{AD}$ and $\mathrm{AMCI}[9,10]$. The total brain volumes for $\mathrm{AD}$ are higher than those previously reported in the Japanese population with a ratio of $966.718-945.899 \mathrm{~cm}^{3}$ [23]. It should be noted that the ratio of women to men is $2.96: 1$ in the Japanese population, whereas in the population in this study it was 2.46:1. It is accepted that the normalized total brain volume is lower in women than in men, a characteristic due to sexual dimorphism [38], thus explaining the lower volume reported in the Japanese population. Another reason that could explain the difference between the volumes is the difference in education levels between the Japanese and Mexican populations: the Mexican population has a level of education of 4.73 years, while in the Japanese population that level is 8.62 years for AD patients. Dementia staging in both populations is mild to moderate according to the MMSE psychometric test; while the Mexican population averaged 16.23 points, the Japanese population averaged 18.87 points. Meanwhile, as for the total brain volume in the aMCI group in the Mexican population, no statistically significant differences were found when compared with the control group $(\mathrm{p}=0.452)$. Conversely, the differences between the normalized total brain volumes were statistically significant between the control group and the AD group $(\mathrm{p}=$ $0.025)$ and between the aMCI group and the AD group ( $p=0.02)$.

\section{Strengths and Weaknesses}

The heterogeneous nature of the population in this study provides a representative sample; demographically and geographically, the sample is representative of the diversity of the Mexican population because subjects were recruited from various social settings, and the educational range allowed for an analysis of a less educated population. By having a significant sample of patients $(n=23)$ with intermediate education and a sufficient sample $(n=14)$ with low education, this study provides information that contributes to the concept of cognitive reserve. Although educational level as a variable was not associated with cognitive impairment, an analysis within each diagnostic group of the hippocampal volume associated with educational level allowed for a thorough understanding of the association between cognitive reserve and educational level. Another advantage of this study is the use of 3.0-tesla MR scanners, given that the contrast between the tissues and the definition of the hippocampal edges in the images is superior to the contrast and definition provided by 1.5-tesla MR scanners used in other studies [21,39]. This improvement in contrast and definition facilitates the manual measurement of the hippocampal formation, providing higher accuracy and less measurement errors [21,40].

The annual hippocampal atrophy rates are of interest to the clinical investigator as they will eventually become part of the diagnostic and prognostic criteria for AD and aMCI. A recent study that evaluated patients from the ADNI reported annual hippocampal atrophy rates of $1.09 \pm 3.0 \%, 2.74 \pm 3.5 \%$ and $4.04 \pm 3.6 \%$ for groups with $\mathrm{NA}$, aMCI and AD, respectively [40]. The accepted annual conversion rate in patients diagnosed with aMCI to AD is $8-19 \%$ [24]. One of the weaknesses of this study is its transversal time sequence. By only making one measurement, it is impossible to calculate the rate of atrophy and thus the conversion rate of aMCI patients to $\mathrm{AD}$; which has significant relevance when predicting the evolution of the dementia syndrome. A selection bias exists because the population is a case series; however, this bias is minimal due to the fact that that the sample is heterogeneous and representative of the Mexican population, having been recruited from approximately a third of the states in Mexico. Another aspect that can enhance the strength of association between cognitive reserve and hippocampal volume in the Mexican population is performing fMRI sequences, such as blood oxygen level dependent (BOLD) with resting paradigm to outline the default mode network (DMN) and changes in activation areas associated with the patients' cognitive decline through time. Incorporating fMRI sequences will allow comparison of the 
Mondragón et al.: Hippocampal Volumetry as a Biomarker for Dementia in People with Low Education

DMN of patients with low and high education, providing a clearer idea about the concept of cognitive reserve in the Mexican population. Finally, the sample could be expanded in order to make associations between handedness, cardiovascular factors and hippocampal volume, as well as to match groups by sex and age.

\section{Conclusion}

When comparing populations with low education, the volumes were within the ranges proposed by previous groups for AD, aMCI and NA [20, 24, 35, 36]. Lower education correlates with lower cognitive reserve; in essence, clinical manifestations of cognitive impairment are present with higher hippocampal volume. The lower the education, the higher the hippocampal volume will be at the patient's clinical cognitive impairment onset, due to a lower cognitive reserve.

Both MMSE and MoCA tests had a good to strong positive correlation with statistically significant differences in the entire population and NA diagnostic group. While the correlation between years of education compared to the volume of the hippocampus was weak to insignificant with no statistically significant differences, both psychometric tests correlated with the hippocampal volume in people with a high educational level (more than 12 years). These two diagnostic tools can be considered as adequate dementia neuropsychological tests in the Mexican population with higher education. The good to strong correlation between both psychometric tests and hippocampal volume in the entire population validates its usefulness in the diagnosis of dementia in a population with a heterogeneous educational level; nonetheless, these diagnostic tools have their limitations when it comes to finding a direct correlation between hippocampal volume and the psychometric tests primarily used to diagnose dementia and $\mathrm{MCI}$ in populations with low and intermediate educational levels.

Cognitive reserve increases an individual's ability to sustain high levels of neuronal injury without the onset of clinical symptoms [9, 37, 38]. This concept provides an explanation to understand the differences in the susceptibility to pathological changes between certain individuals; while some are capable of withstanding these changes maintaining an adequate function, others are not $[37,38]$. Cognitive reserve is linked to neuroplasticity and is influenced by genetic and epigenetic factors, such as environment and education [41]; however, these associations need to be explored in a near future. The concept of cognitive reserve represents the ability to activate alternate neural networks or cognitive strategies to cope with this besieging pathology [9]. Although patients with greater cognitive reserve can tolerate greater pathological burden, they deteriorate faster when cognitive impairment symptoms manifest [37].

PET and fMRI are helpful to delineate the DMN and hence permit a qualitative measure of cognitive reserve [37, 42]. Cognitive reserve is preferably studied through fMRI sequences, through the study of DMNs, obtained by BOLD sequences $[43,44]$. DMN is closely associated with episodic memory processing [43]. The calculated sensitivity and specificity for changes in the DMN to distinguish between AD patients and healthy subjects are 85 and 75\%, respectively [43]; nonetheless, if the volume of interest and independent component analysis evaluation techniques in the BOLD sequence are employed jointly, the sensitivity and specificity are 100 and 95\%, respectively [44]. While considering the concept of cognitive reserve as a biomarker is premature [37], the future of cognitive decline assessment steers to tests that measure functional injury rather than structural damage; nonetheless, these diagnostic tools have a complementary value. 
Mondragón et al.: Hippocampal Volumetry as a Biomarker for Dementia in People with Low Education

\section{Acknowledgements}

We appreciate the collaboration of Fernando Alejandro Barrios-Álvarez, PhD, Juan Fernández Ruiz, PhD, Edgar Fernando Gutiérrez-Vela, MD, Omar Jiménez-Zarazúa, MD, Juan José Ortiz-Retana, MS, Erick Humberto Pasaye-Alcaraz, radiographers Sergio Oswaldo Sánchez-Corona and Jorge Octavio Bravo-Gamboa, and nurse Alejandra Arenas-Tecuapetla for their support in the completion of this project.

\section{Statement of Ethics}

All patients or their primary caretaker provided their signed informed consent form before undergoing the MRI scan, medical interview and psychometric tests. The study was approved by the bioethics committee of Instituto de Neurobiología, UNAM.

\section{Disclosure Statement}

The authors do not report any conflict of interest.

\section{References}

$>1$ Wimo A, Jönsson L, Bond J, et al: The worldwide economic impact of dementia, 2010. Alzheimers Dement 2013;9:1-11.

2 Sosa-Ortiz A, Acosta-Castillo I, Prince MJ: Epidemiology of dementias and Alzheimer's disease. Arch Med Res 2012;43:600-608.

3 World Health Organization: Global Burden of Disease 2004 Update. Geneva, World Health Organization, 2008.

4 Prince M, Bryce R, Alnanese E, et al: The global prevalence of dementia: a systematic review and metaanalysis. Alzheimers Dement 2013;9:63-75.

5 Hebert LE, Weuve J, Scherr PA, et al: Alzheimer disease in the United States (2010-2050) estimated using the 2010 census. Neurology 2013;80:1778-1783.

6 Alzheimer Disease International: World Alzheimer Report 2009. London, Alzheimer Disease International, 2009.

7 Dubois B, Feldman HH, Jacova C, et al: Research criteria for diagnosis of Alzheimer's disease: revising the NINCDS-ADRDA criteria. Lancet Neurol 2007;6:734-746.

8 McKhann GM, Knopman DS, Chertkow H, et al: The diagnosis of dementia due to Alzheimer's disease: recommendations from the National Institute on Aging-Alzheimer's Association workgroups on diagnostic guidelines for Alzheimer's disease. Alzheimers Dement 2011;7:263-269.

-9 Sperling RA, Aisen PS, Beckett LA, et al: Toward defining the preclinical stages of Alzheimer's disease: recommendations from the National Institute on Aging-Alzheimer's Association workgroups on diagnostic guidelines for Alzheimer's disease. Alzheimers Dement 2011;7:280-292.

$>10$ Albert MS, DeKosky ST, Dickson D, et al: The diagnosis of mild cognitive impairment due to Alzheimer's disease: recommendations from the National Institute on Aging-Alzheimer's Association workgroups on diagnostic guidelines for Alzheimer's disease. Alzheimers Dement 2011;7:270-279.

11 Jack CR Jr, Albert MS, Knopman DS, et al: Introduction to the recommendations from the National Institute on Aging Association workgroups on diagnostic guidelines for Alzheimer's disease. Alzheimers Dement 2011;7: 257-262.

12 Petersen RC, Doody R, Kurz A, et al: Current concepts in mild cognitive impairment. Arch Neurol 2001;58: 1985-1992.

13 de Jaeger C: Physiologie du vieillissement. EM Consulte DOI 10.1016/S1283-0887(11)56633-2.

14 Jack CR Jr, Knopman DS, Jagust WJ, et al: Tracking pathophysiological process in Alzheimer's disease: an updated hypothetical model of dynamic biomarkers. Lancet Neurol 2013;12:207-216.

15 Folstein MF, Folstein SE, McHugh PR: 'Mini-mental state': A practical method for grading the cognitive state of patients for the clinician. J Psychiatr Res 1975;12:189-198.

16 Nasreddine ZS, Phillips NA, Bédirian V, et al: The Montreal Cognitive Assessment, MoCA: a brief screening tool for mild cognitive impairment. J Am Geriatr Soc 2005;53:695-699. 
Duvernoy HM: Sectional Anatomy and Magnetic Resonance Imaging; in Duvernoy HM: The Human Hippocampus, ed 3. Berlin, Springer, 2005, chapter 7, pp 129-217.

18 Luby M, Spencer DD, Kim JH, et al: Hippocampal MRI volumetrics and temporal lobe substrates in medial temporal lobe epilepsy. Magn Reson Imaging 1995;13:1065-1071.

19 Jack CR Jr, Theodore WH, Cook M, et al: MRI-based hippocampal volumetrics: data acquisition, normal ranges, and optimal protocol. Magn Reson Imaging 1995;13:1057-1064.

20 Csernansky JG, Wang L, Swank J, et al: Preclinical detection of Alzheimer's disease: hippocampal shape and volume predict dementia onset in the elderly. Neuroimage 2005;25:783-792.

21 Pruessner JC, Li LW, Serles W, et al: Volumetry of hippocampal and amygdala with high-resolution MRI and three-dimensional analysis software: minimizing the discrepancies between laboratories. Cereb Cortex 2000; 10:433-442.

22 Jeukens CR, Vlooswijk MC, Majoie HJ, et al: Hippocampal MRI volumetry at 3 tesla. Invest Radiol 2009;44: 509-517.

23 Winterburn JL, Pruessner JC, Chavez S, et al: A novel in vivo atlas of hippocampal subfields using high-resolution 3 T magnetic resonance imaging. Neuroimage 2013;74:254-265.

24 Yasuda M, Mori E, Kitagaki H, et al: Apolipoprotein E epsilon 4 allele and whole brain atrophy in late-onset Alzheimer's disease. Am J Psychiatry 1998;155:779-784.

25 Colliot 0, Chételat G, Chupin M, et al: Discriminating between Alzheimer disease, mild cognitive impairment, and normal aging by using automated segmentation of the hippocampus. Radiology 2008;248:194-201.

26 Chincarini A, Bosco P, Calvini P, et al: Local MRI analysis approach in the diagnosis of early and prodromal Alzheimer's disease. Neuroimage 2011;58:469-480.

27 Devanand DP, Bansal R, Liu J, et al: MRI hippocampal and entorhinal cortex mapping in predicting conversion to Alzheimer's disease. Neuroimage 2012;60:1622-1629.

28 Velayudhan L, Proitsi P, Westman E, et al: Entorhinal cortex thickness predicts cognitive decline in Alzheimer's disease. J Alzheimers Dis 2013;33:755-766.

29 Albert M, DeCarli C, DeKosky S, et al: The Use of MRI and PET for Clinical Diagnosis of Dementia and Investigation of Cognitive Impairment: A Consensus Report. San Francisco, Alzheimer's Association, 2004.

-30 Chupin M, Mukuna-Bantumbakulu AR, Hasboun D, et al: Anatomically constrained region deformation for automated segmentation of the hippocampus and the amygdala: method and validation on controls and patients with Alzheimer's disease. Neuroimage 2007;34:996-1019.

-31 Jack CR Jr, Twomey CK, Zinsmeister AR, et al: Anterior temporal lobes and hippocampal formations: normative volumetric measurements from MR images in young adults. Radiology 1989;172:549-554.

32 Schmidt R, Havas D, Ropele S, et al: MRI in dementia. Neurol Clin 2008;27:221-236.

-33 Boccardi M, Bocchetta M, Ganzola R, et al: Operationalizing protocol differences for EADC-ADNI manual hippocampal segmentation. Alzheimers Dement 2015;11:184-194.

-34 Apostolova LG, Thompson PM: Mapping progressive brain structural changes in early Alzheimer's disease and mild cognitive impairment. Neuropsychologia 2008;46:1597-1612.

35 Yavuz BB, Ariogul S, Cankurtaran M, et al: Hippocampal atrophy correlates with the severity of cognitive decline. Int Psychogeriatr 2007;19:767-777.

-36 Stern Y: Cognitive reserve in ageing and Alzheimer's disease. Lancet Neurol 2012;11:1006-1012.

37 Steffener J, Stern Y: Exploring the neural basis of cognitive reserve in aging. Biochim Biophys Acta 2012;1822: 467-473.

38 Skup M, Zhu H, Wang Y, et al: Sex differences in grey matter atrophy patterns among AD and aMCI patients: results from ADNI. Neuroimage 2011;56:890-906.

-39 Briellmann RS, Syngeniotis A, Jackson GD: Comparison of hippocampal volumetry at 1.5 tesla and at 3 tesla. Epilepsia 2001;42:1021-1024.

40 Cardoso MJ, Leung K, Modat M, et al: STEPS: similarity and truth estimation for propagated segmentations and its application to hippocampal segmentation and brain parcelation. Med Image Anal 2013;17:671-684.

-41 Vasile C: Cognitive reserve and cortical plasticity. Proc Soc Behav Sci 2013;78:601-604.

42 Greicius MD, Srivastava G, Reiss AL, et al: Default-mode network activity distinguishes Alzheimer's disease from healthy aging: evidence from functional MRI. Proc Natl Acad Sci USA 2004;101:4637-4642.

43 Shin J, Kepe V, Small GW, et al: Multimodal imaging of Alzheimer pathophysiology in the brain's default mode network. Int J Alzheimers Dis 2011;2011:687945.

44 Koch W, Teipel S, Mueller S, et al: Diagnostic power of default mode network resting state fMRI in the detection of Alzheimer's disease. Neurobiol Aging 2012;33:466-478. 